\title{
Creating a Mathematical Museum on Your Desk
}

\author{
George W. Hart \\ Stony Brook University \\ Department of Computer Science
}

Travel is unnecessary to see certain objects of mathematical interest, as new technology makes it convenient to be a stay-at-home explorer of 3D mathematical models. With the right equipment, one can create and enjoy a mathematical museum collection without leaving ones office. Figure 1 shows an assortment of physical models on shelves of my office. New technologies-commercially available or under development-allow the creation of physical models that are very compact, intricate, and accurate. These state-of-the-art processes involve the automated (robotic) construction of physical objects by assembling thin cross-sections calculated from the designer's computer file describing the structure to be built. Engineers call these "rapid prototyping" fabrication processes because at present their major application is for quickly making prototypes of parts that are later mass-produced by industrial techniques. However, the more general term "solid freeform fabrication" (SFF) conveys the idea that these objects need be neither rapid—some of these models took a machine three days to build—nor prototypes—for us they are the final result.

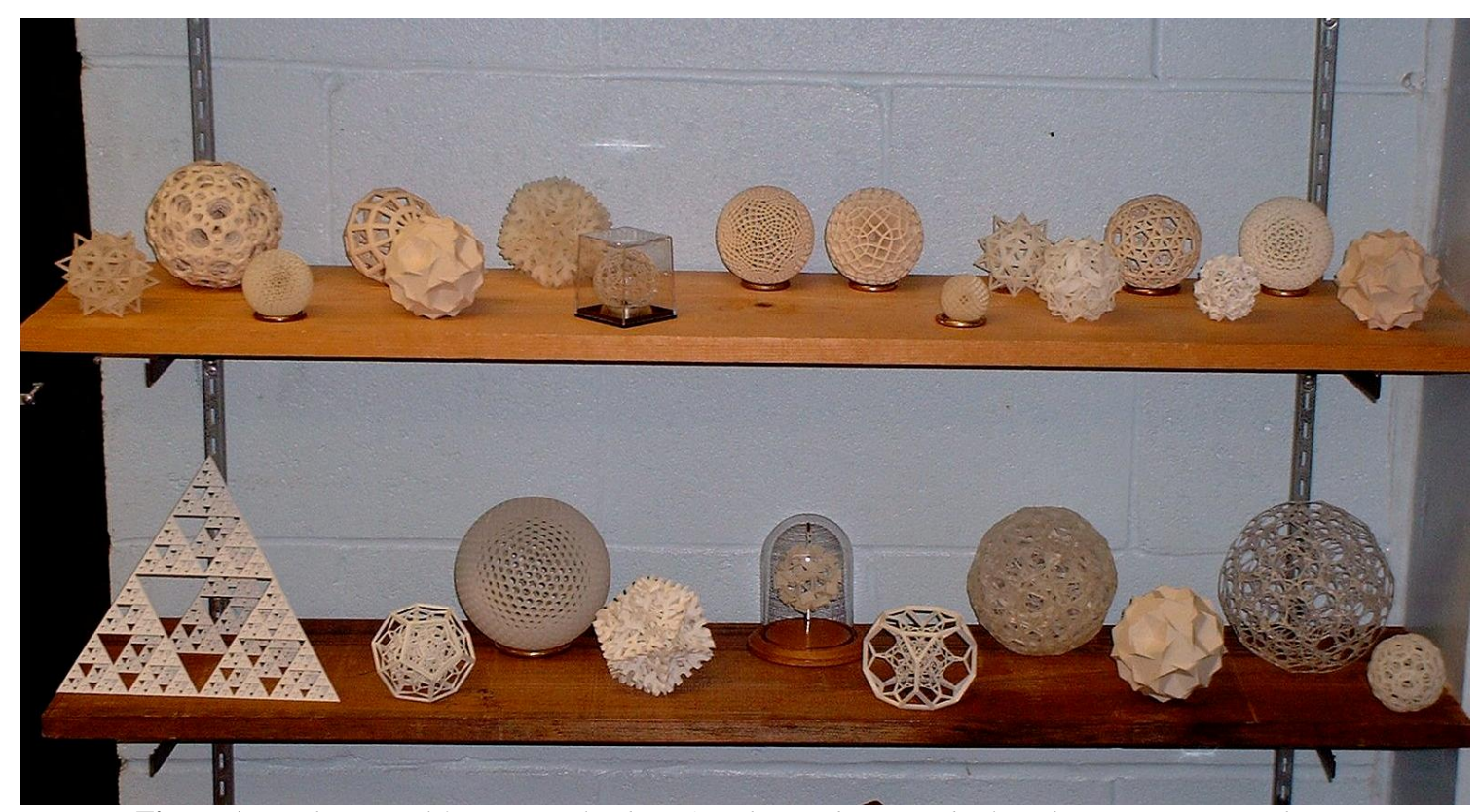

Figure 1. Mathematical forms, mostly about 3 inches in diameter, built with various SFF machines.

SFF technology has applications in the creation of all types of educational 3D objects, e.g., topological, algebraic, molecular, crystal, anatomical, or historical models. Remnants of older collections, e.g., at Gottingen and Arizona University, speak to us from another era, when models were of considerable didactic importance. In the late nineteenth century, plaster, wood, wire, or cardboard mathematical models were promoted by prominent mathematicians such as Felix Klein. Their use diminished in the twentieth century, but many mathematicians consider models valuable for building intuition and for communicating mathematical ideas to students and the public. A "Cabinet of Curiosities" featuring mathematical forms can serve to inspire non-mathematicians and hook students. Nothing can substitute for the visual and tactile pleasure of handling a model, spinning it in ones hand, comparing it to another model in the other hand, etc. This paper illustrates only geometric models, but the references include links to algebraic surface models by Carlo Sequin or Jonathan Chertok that were generated in a similar spirit. 


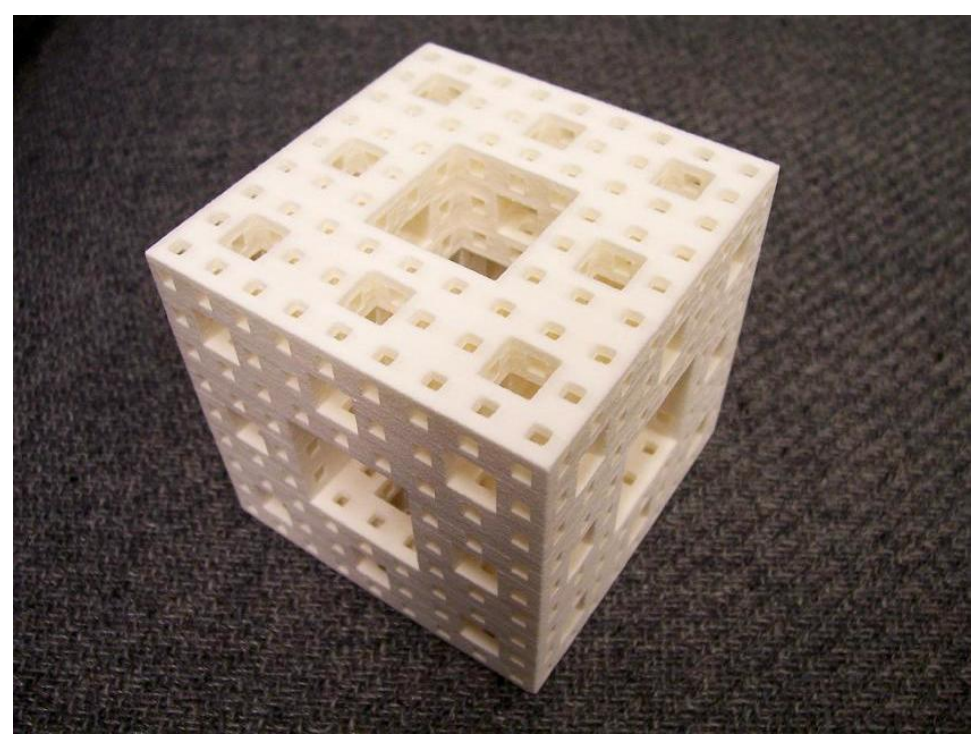

Figure 2. Model of Menger Sponge, 3 inches.

Fractals, because they are intricate and procedurally generated, are natural subjects for SFF. Figure 2 shows a model of a well-known example, the Menger Sponge. This fractal is (in the limit) all surface with no volume, and a model makes this clear by its lightness when picked up. The model in Figure 2 is made of nylon by a SFF process called "Selective Laser Sintering" (SLS) in which a high-power computercontrolled laser fuses nylon dust in just the places where we want solidity and leaves the dust to be vacuumed away in the places where we want voids.

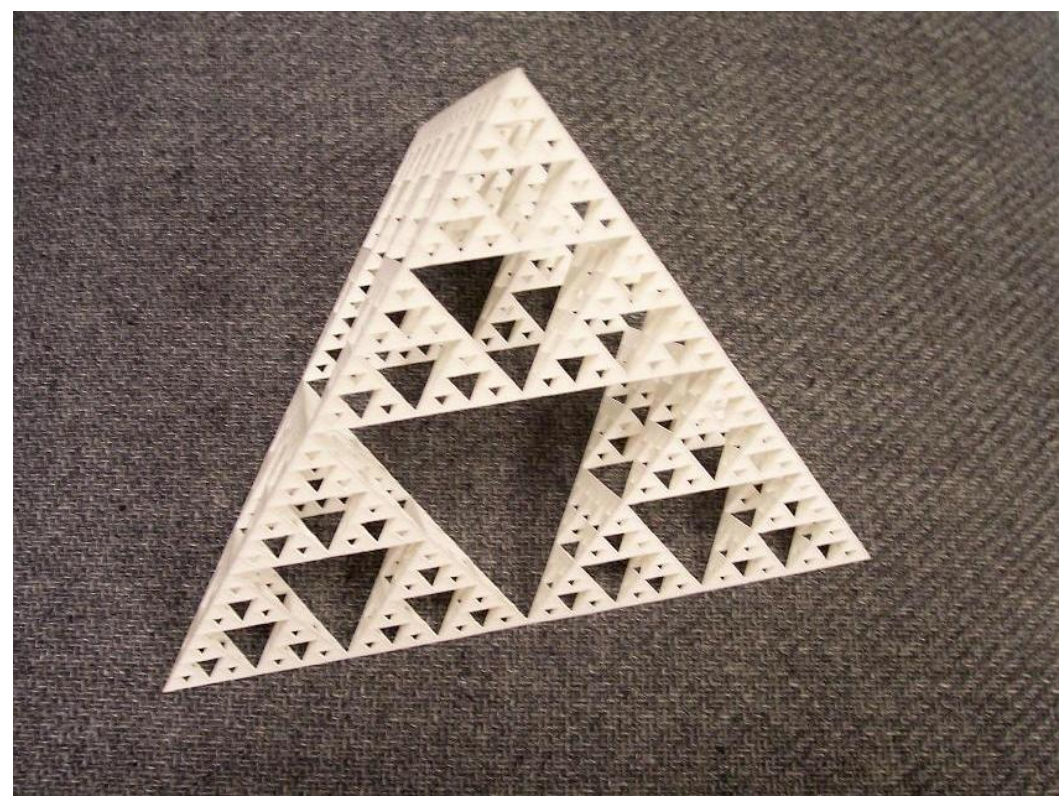

Figure 3. Model of Sierpinski Tetrahedron, 8 inch.

Another familiar fractal, the "Sierpinski Tetrahedron", is shown in Figure 3. This model is also made of nylon by the SLS process. Underlying all SFF models are digital descriptions of the forms to be constructed. A file describing the boundary surface as a triangulated manifold must be created as input for guiding the SFF machine. With some effort, one may adapt commercial Computer Aided Design packages (CAD software designed for engineers) to create the geometry description files. However, my interest is in writing special-purpose software that conveniently generates families of interesting objects. 


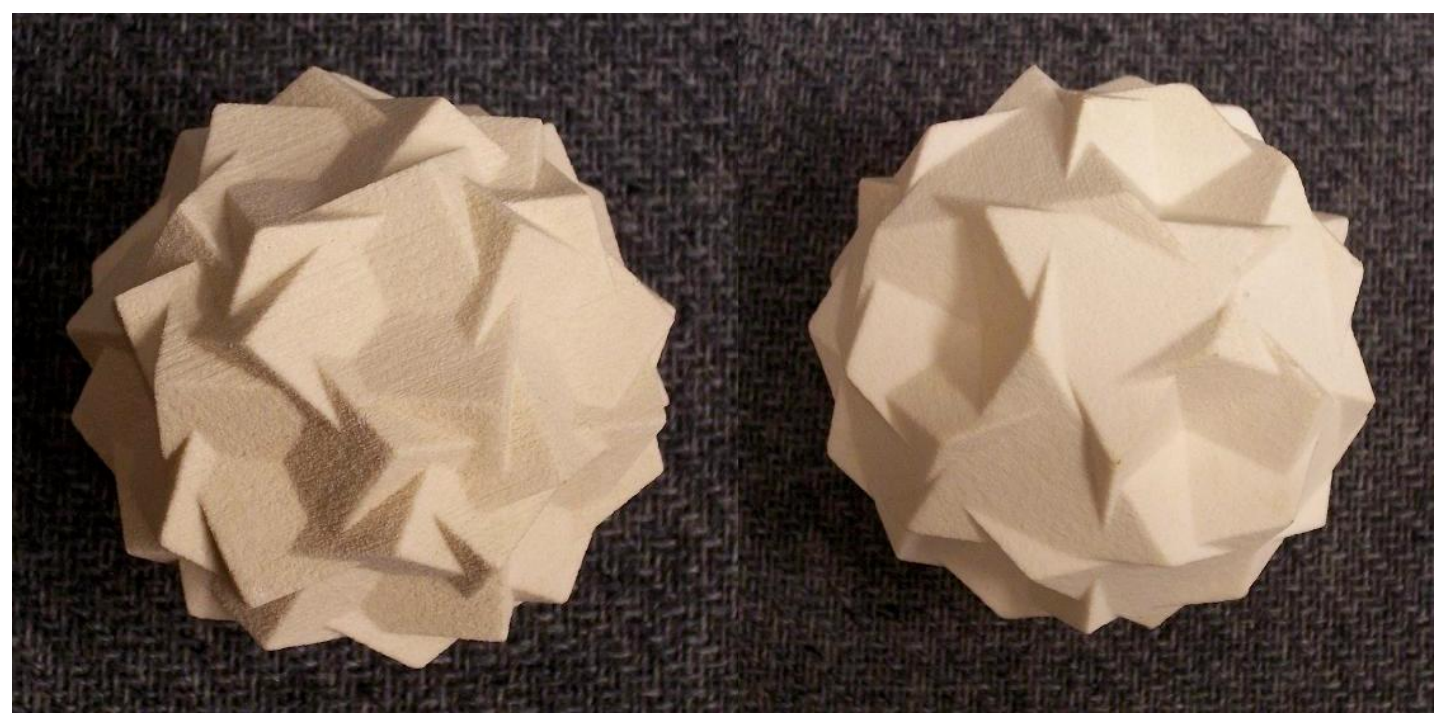

Figure 4. Compounds: Six pentagonal prisms, Five truncated tetrahedra, 3 inch diam.

Figure 4 shows models of two beautiful uniform polyhedral compounds with icosahedral symmetry. The first is six concentric pentagonal prisms; the second is five concentric truncated tetrahedra. First described in the mathematics literature by John Skilling in 1976, I do not know of anyone making a physical model of either before I made these in 1999. These are made of plaster by the "3D Printing" SFF method, which uses inkjet printer technology to squirt water selectively in the places where the plaster dust is to be hardened, leaving the unmoistened plaster dust to be vacuumed away.

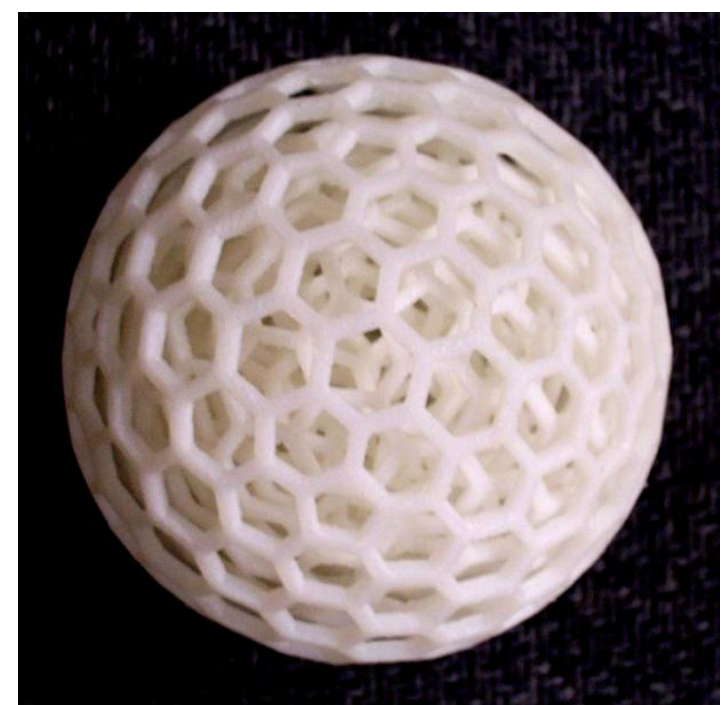

Figure 5. Seven nested Goldberg polyhedra.

The object in Figure 5 is composed of seven different nested polyhedra, each free to rotate independently of the others. Each layer is a polyhedron with twelve pentagons and some number of hexagons, arranged with icosahedral symmetry. These are the examples with 42 to 192 faces from an infinite family of such forms described by Michael Goldberg in the 1930's. Goldberg described their topology and I have explored various ways to realize them geometrically with planar faces. The model consists of only their edges, so one can see through the face openings into the progressively smaller inner polyhedra. This model is made of ABS plastic by a SFF technique called "Fused Deposition Modeling" (FDM). It works essentially like a hot-melt glue gun on a robot arm, squirting molten plastic that cools to make the solid model. 


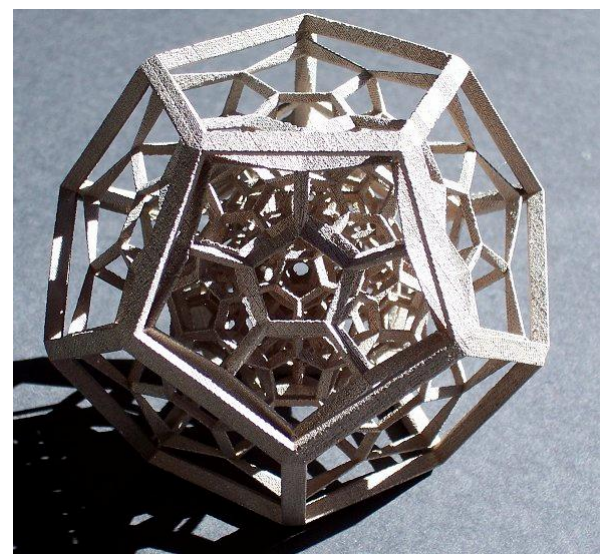

Figure 6. Shadow of 4D 120-cell, 3 inches.

The 120-cell is a regular four-dimensional polytope composed of 120 regular dodecahedra, three around each edge, discovered by Ludwig Schläfli in the 1850's. Figure 6 shows a three-dimensional shadow of the 120-cell, via a perspective transformation, which results in 119 progressively flattened dodecahedra packed into an outer regular dodecahedron. Wire and thread models of this form were designed by Victor Schlegel and sold commercially in the 1880's through catalogs of mathematical models. Here I have recreated the form and had it fabricated of sintered stainless steel infused with bronze by the "Prometal" SFF process.

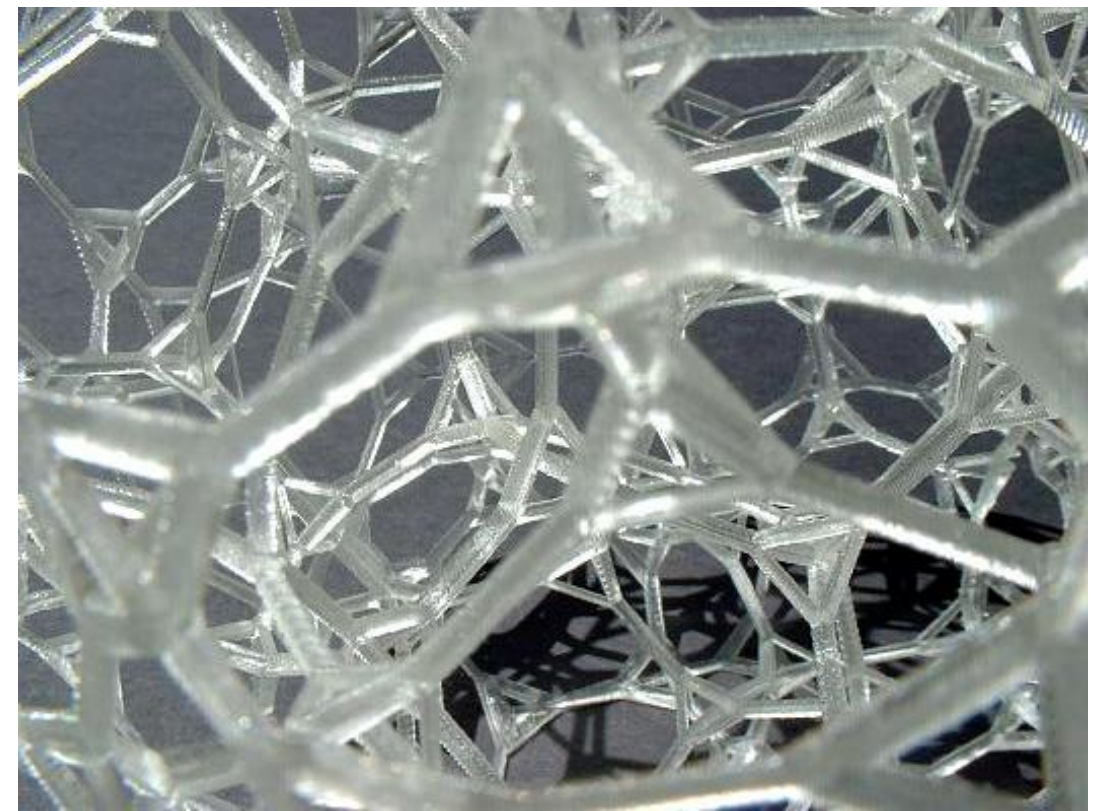

Figure 7. Shadow of $4 D$ truncated 120-cell, 6 inches, detail.

The "truncated 120-cell" is a more complex four-dimensional polytope, analogous to an Archimedean polyhedron. First described in a 1910 paper by Alicia Boole Stott, it consists of 120 truncated dodecahedra and 600 regular tetrahedra. They are arranged so that the symmetry group is transitive on its 2400 vertices, each equivalently surrounded by three truncated dodecahedra and one tetrahedron. It can be projected via an orthogonal transformation to the intricate 3D form shown in close-up in Figure 7. The 4D "front" and "back" overlay, halving its 3D complexity, which is helpful when one is learning to visualize such forms. This model is made of an epoxy polymer by the "stereolithography" SFF process. A computer-controlled ultraviolet laser is used to catalyze the hardening of a liquid photopolymer in just the places where one wants solidity. 
In summary, new SFF technologies allow the fabrication of mathematical models that would be very difficult to produce by any other means. I find a great pleasure in handling a real 3D object that I receive from these machines after previously visualizing it, designing its coordinates, and only viewing it on the computer screen. Although relatively expensive at present, SFF fabrication costs are decreasing as the technology develops. Currently, machines are generally available at manufacturing design companies, at research universities, and at commercial "rapid prototyping" service bureaus. Files for the above objects are freely available on my web site and can be sent to a variety of machines for fabrication. In the future, I expect all schools will have SFF capability and textbook publishers and other educational sources will provide files and software. Teachers will fabricate what is relevant to their classes and pass the models around the room for students to examine. Anyone will be able to create a "cabinet of curiosities" which can excite a sense of wonder about mathematics.

\section{References:}

A) Mathematical models:

- Gerd (Gerhard) Fischer, Mathematische Modelle, (Plates) and Mathematical Models: From the Collections of Universities and Museums (English Commentary), Vieweg, Braunschweig, 1986.

- Peggy Kidwell, "American Mathematics Viewed Objectively: The Case of Geometric Models," in Vita Mathematica: Historical Research and Integration with Teaching, Ron Calinger ed., MAA, 1996, pp. 197-208.

- William Mueller, "Mathematical Wunderkammern”, American Mathematical Monthly, 108, November 2001, pp. 785-796.

- Angela Vierling, list of online model collections: http://www.math.harvard.edu/ angelavc/models/locations.html

B) Mathematical sources for the illustrated objects:

- George W. Hart, SFF files, http://www.georgehart.com/rp/rp.html

- H.S.M. Coxeter, Regular Polytopes, 1963, (Dover reprint, 1973).

- Michael Goldberg, "A Class of Multi-Symmetric Polyhedra," Tohoku Mathematics Journal, 43, 1937, pp. 104-108.

- $\quad$ Benoît Mandelbrot, The Fractal Geometry of Nature. Freeman, 1982.

- J. Skilling, "Uniform Compounds of Uniform Polyhedra," Mathematical Proceedings of the Cambridge Philosophical Society, Vol. 79, pp. 447-457, 1976.

- Alicia Boole Stott, "Geometrical deduction of semiregular from regular polytopes and space fillings," Verhandelingen der Koninklijke Akademie van Wetenschappen te Amsterdam, (eerste sectie), Vol. 11, No. 1, pp. 1-24 plus 3 plates, 1910.

C) SFF Manufacturers:

- Bathsheba Grossman, http://www.bathsheba.com/math/

- 3D Systems, http://www.3dsystems.com/

- Extrude Hone Prometal, http://www.prometal-rt.com/

- Stratasys Corporation, http://www.stratasys.com/

- Z Corporation, http://www.zcorp.com/

D) Details of generating SFF files for mathematical forms:

- G. Hart, "Solid-Segment Sculptures," Proceedings of Colloquium on Math and Arts, Maubeuge, France, 20-22 Sept. 2000, and in Mathematics and Art, Claude Brute ed., Springer-Verlag, 2002.

- George W. Hart, "Rapid Prototyping of Geometric Models," Proceedings of Canadian Conference on Computational Geometry, August 2001.

- $\quad$ George W. Hart, "In the Palm of Leonardo's Hand," Nexus Network Journal, vol. 4, no. 2, Spring 2002; reprinted in Symmetry: Culture and Science, vol. 11, 2000 (appeared 2003), pp. 17-25.

- George W. Hart "4D Polytope Projection Models by 3D Printing", to appear in Hyperspace.

- Jonathan Chertok, http://www.oliverlabs.net/

- Carlo Sequin, http://www.cs.berkeley.edu/ sequin/ 